\title{
Preface Issue 2-2015
}

\author{
Hans-Christoph Grunau ${ }^{1}$
}

(C) Deutsche Mathematiker-Vereinigung and Springer-Verlag Berlin Heidelberg 2015

Friedrich Hirzebruch, who died in 2012 at the age of 84, was one of the most important German mathematicians of the second half of the twentieth century. This applies not only to his mathematical achievements - mainly in topology, geometry and number theory - and his huge school of at least 52 Ph.D. students. He also made enormous efforts and had great success in reshaping mathematics in Germany after the Second World War. The Sonderforschungsbereich Theoretische Mathematik, the Arbeitstagung and the Max-Planck-Institut für Mathematik played an important role in making Germany once again a place which mathematicians from all over the world would like to visit. The Deutsche Mathematiker-Vereinigung (DMV) owes a particularly deep gratitude to Friedrich Hirzebruch who served twice as its chairman, in 1962 and 1990. In 1962, one year after the wall was built, the Gesellschaft für Mathematik der DDR was founded and urged to branch off from the DMV. One purpose of his second election in 1990 was that he should chair the reunification of both societies and to celebrate at the same time the 100th anniversary of the DMV. In the 28 years in between he ensured that the ties to the East were not severed. One of his best known former Ph.D.-students, Don Zagier, gives a fairly detailed picture of all these and many more facets of Friedrich Hirzebruch's life within mathematics.

Often, pictures are available only in a corrupted form and one has to try to reconstruct the original ones. One may think for instance of a real valued function describing the gray-value of the picture at the actual position. If one assumes that the corruption mechanism (the corruption mapping) is known up to some "noise" one has to seek a preimage of the corrupted picture under this mapping. In principle one could apply the corruption mapping to all possible pictures and search for those

H.-Ch. Grunau

hans-christoph.grunau@ovgu.de

1 Institut für Analysis und Numerik, Fakultät für Mathematik, Otto-von-Guericke-Universität,

Postfach 4120, 39016 Magdeburg, Germany 
whose images minimise a suitable distance functional from the given corrupted picture. Due to the "bad" properties of the corruption mapping this problem is in this form not well posed. In order to resolve this issue, one adds a regularisation term containing first and second order derivatives of the unknown preimage to the distance functional. Gabriele Steidl explains in her survey article on "Combined first and second order variational approaches for image processing" the recent development of such techniques, and illustrates them by two rather different applications.

Functions of bounded variation and related function spaces are fundamental for Gabriele Steidl's survey article and are also the topic of a recent book by Jürgen Appell, Józef Banas, and Nelson José Merentes Díaz, which is reviewed by Michel Willem. The second review concerns a book by Luis Barreira and Yakov Pesin on smooth ergodic theory and is provided by Gerhard Knieper. 